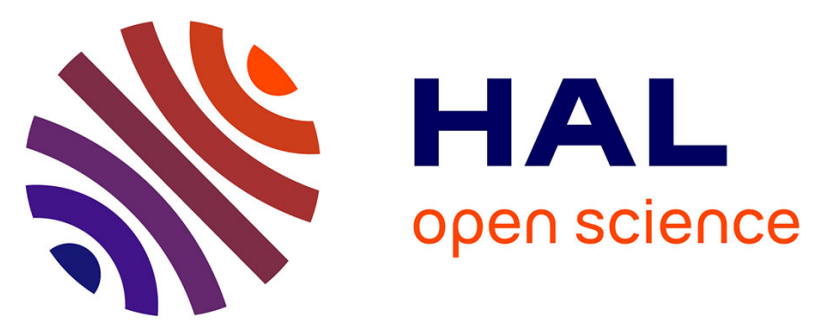

\title{
Photorefractive beam-steering system that uses energy transfer in a BaTiO3 crystal for a fiber-array interconnect
}

Pierre Mathey, Raymond Mercier, Gilles Pauliat, Gérald Roosen, Philippe Gravey

\section{To cite this version:}

Pierre Mathey, Raymond Mercier, Gilles Pauliat, Gérald Roosen, Philippe Gravey. Photorefractive beam-steering system that uses energy transfer in a BaTiO3 crystal for a fiber-array interconnect. Applied optics, 1995, 34 (35), pp.8220-8229. hal-00856691

\section{HAL Id: hal-00856691 \\ https://hal-iogs.archives-ouvertes.fr/hal-00856691}

Submitted on 2 Sep 2013

HAL is a multi-disciplinary open access archive for the deposit and dissemination of scientific research documents, whether they are published or not. The documents may come from teaching and research institutions in France or abroad, or from public or private research centers.
L'archive ouverte pluridisciplinaire HAL, est destinée au dépôt et à la diffusion de documents scientifiques de niveau recherche, publiés ou non, émanant des établissements d'enseignement et de recherche français ou étrangers, des laboratoires publics ou privés. 


\title{
Photorefractive beam-steering system that uses energy transfer in a $\mathrm{BaTiO}_{3}$ crystal for a fiber-array interconnect
}

\author{
P. Mathey, R. Mercier, G. Pauliat, G. Roosen, and Ph. Gravey
}

\begin{abstract}
A beam-control system to write gratings on a holographic plane is studied. The arrangement is designed to interconnect two 1024 monomode fiber arrays. The beam-control system is composed of two subsystems: a beam steerer, which deflects one incident beam toward 1024 positions, and a collimating system, which adapts the shape of the deflected beam to the holographic plane. The collimating system was studied only after the beam steerer had been fully built and tested. It is based on the photorefractive amplification of a beamlet selected by a shutter array. The deflection efficiency is enhanced by a factor 1500 with the photorefractive crystal, and the signal-to-noise ratio is larger than 5500. The influence of the optical aberrations on the coupling losses of the infrared beams in the monomode fibers are evaluated.

Key words: Optical interconnections, beam steering, microlens array, photorefractive amplifiers, signal-to-noise ratio. $\odot 1995$ Optical Society of America
\end{abstract}

\section{Introduction}

Reconfigurable optical free-space interconnection systems are being actively studied for various applications in photonic switching and interconnects. The roles of these systems are to route signals issued from an array of emitters toward an array of receivers. In telecommunication applications in which high-bitrate signals (above $1 \mathrm{Gbyte} / \mathrm{s}$ ) are manipulated, it is very desirable to interconnect arrays of single-mode fibers. This could, for instance, permit the realization of large-scale optical cross-connect systems and to cascade them by the insertion of optical amplifiers. Different free-space interconnection systems have

When this work was performed P. Mathey, R. Mercier, G. Pauliat, and G. Roosen were with the Institut d'Optique Théorique et Appliquée, Unité de Recherche14Associée au Centre National dela Recherche Scientifique, Centre Scientifique d'Orsay, Bât. 503, Boite Postale 147, 91403 Orsay Cedex, France; and Ph. Gravey was with France Télécom, Centre National d' Études des Télécommunications, Lannion B, Réseaux Intégrés Optiques, Processeurs de Commutation Optique, Route de Trégastel, Boite Postale 40, 22301 Lannion Cedex, France.

P. Mathey is now with the Université de Bourgogne, Laboratoire de Physique, Matériaux pour l'Optique, Unité de Recherche 1796 Associée au Centre National de la Recherche Scientifique, Bât. Mirande, Boite Postale 138, 21004 Dijon Cedex, France.

Received 7 December 1994; revised manuscript received 31 J uly 1995.

0003-6935/95/358220-10\$06.00/0.

(c) 1995 Optical Society of America. been proposed. A first architecture is based on the Stanford matrix-vector multiplier, ${ }^{1}$ which broadcasts power from all inputs to all outputs, and outputs that are not connected to a particular input are shadowed from it by a spatial light modulator (SLM). This shadowing yields a power loss, which increases with the number of ports in the system, especially when single-mode fibers are used as output receivers. In principle, using beam deflection avoi ds this drawback because all the power is directed toward the desired output if the deflection efficiency is high enough. The deflection between one input array and one output array can be realized if several arrays of beam shifters are cascaded, ${ }^{2}$ or if reconfigurable hol ograms are used that are written either on an electrically addressed SLM or on an optically addressed SLM. An optically addressed SLM provides the highest resolution and seems to be best suited for routing large numbers of ports (approximately 1000). Some experimental demonstrations of reconfigurable interconnections by means of hologram arrays have already been presented.3,4 Because all these systems use a one-hologram array, the direction of the beam arriving at the output receiver depends on the inputport position. This setup is not compatible with single-mode output fibers; for that case, a possible solution is to use a second hologram array, which redirects the deflected beams to a unique direction that is common to all the output ports (i.e., a twodeflection-stage system). 
We present here a study of an optical addressing system for a 1024-hol ogram array; the characteristics of this addressing system have been designed to be compatible with the interconnection of two 1024 single-mode-fiber arrays. Particular attention has been devoted to the aberrations introduced by the addressing beams because this point is critical to the achievement of efficient coupling of the deflected beams into single-mode fibers. The system is designed to write reconfigurable gratings in an holographic plane, $\mathrm{P}_{\mathrm{c}}$. This holographic plane is composed of $\mathrm{N} \times \mathrm{N}=\mathrm{N}^{2}$ juxtaposed elementary cells. In each cell, an elementary grating is written, and it diffracts a collimated beam (at $\lambda=1.33 \mu \mathrm{m}$ ) issued from a monomode fiber of an emission array of $\mathrm{N} \times$ $\mathrm{N}=\mathrm{N}^{2}$ fibers, toward a single monomode fiber of an $\mathrm{N} \times \mathrm{N}=\mathrm{N}^{2}$ fiber reception array (see Fig. 1). Updating the gratings reconfigures the interconnections between the two arrays. These gratings are written by means of the interference of two writing beams: one that has a fixed incidence, and one whose direction is adjusted according to the grating that must be written. The holographic plane is made from a photothermoplastic material. ${ }^{5}$ Each cell of this plane can be sensitized by a corona discharge and local heating. The writing beams cover the entire holographic plane, and only the sensitized cell is reconfigured. The gratings arethus written sequentially but read in parallel. The reception fiber to be interconnected can be chosen by a change in the fringe spacing of the elementary grating.
The wavel engths of the two writing beams are $\lambda=$ $514 \mathrm{~nm}$. The sensitivity of the photothermoplastic material is highest for this wavel ength, but it must be noted that all the characteristics of the components of the system can be recalculated for any other wavelength. The complete beam-control system is made from the association of two subsystems: first a beam steerer $^{6}$ (see Subsection 2.B.) that permits the variation of the incident angle of the moveable writing beam, and second a system that adapts the characteristics of the beam issued from the beam steerer to the holographic plane. This second system (see Subsection 2.C.), called the collimating system, also shapes the second fixed writing beam. In the following paper, the design (Section 2) and the characterization (Section 3) of the beam-control system are described.

\section{System Design}

A. Requirements

The design of the beam-control system depends on certain required performances of the interconnect system, as follows:

(1) A surface $E^{2}=E \times E=100 \mathrm{~cm}^{2}$ of the photothermoplastic plane that is required to interconnect two square arrays of $\mathrm{N} \times \mathrm{N}=1024$ fibers.

(2) The switching time $\tau$ must be approximately $50 \mathrm{~ms}$,

(3) The spatial frequencies $1 / \Lambda$ of the gratings must be larger than 200 lines/ $\mathrm{mm}$ to keep a high

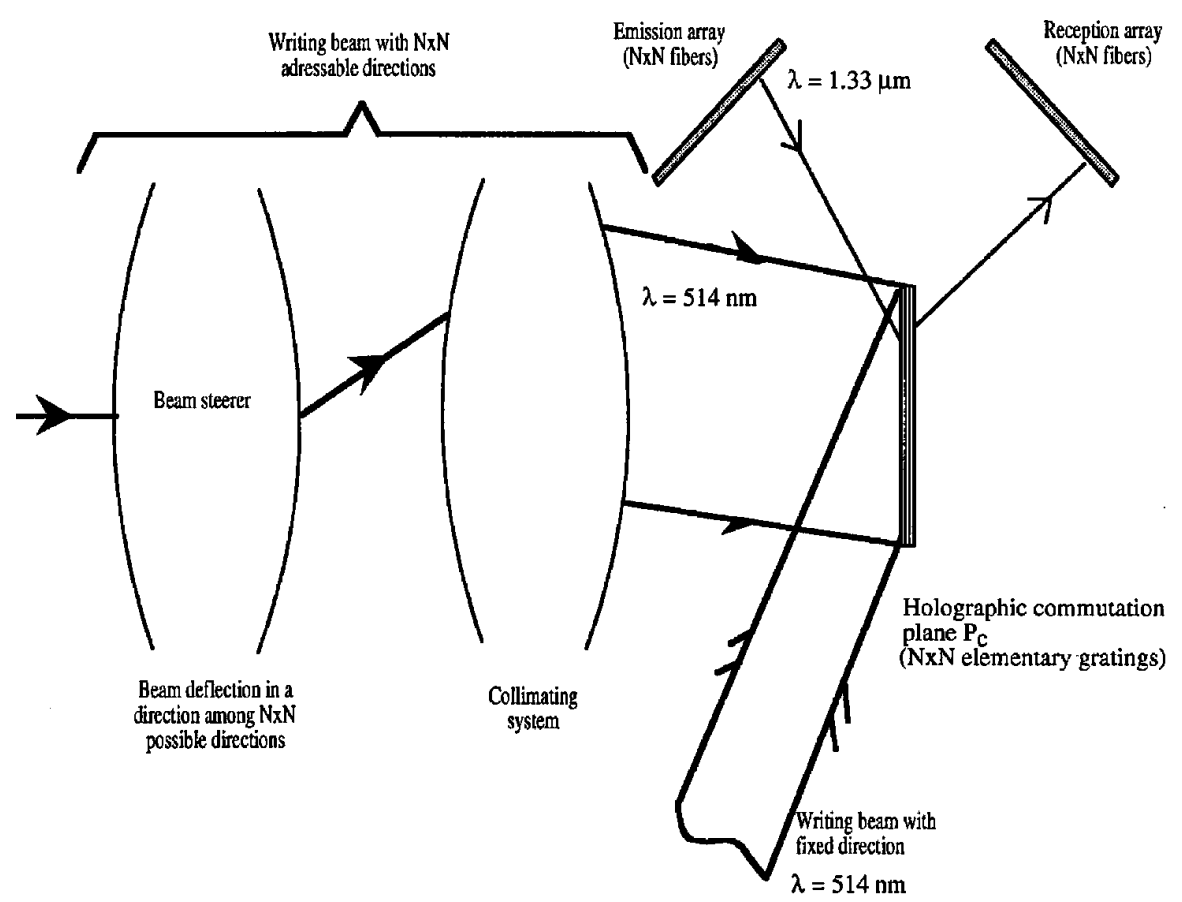

Fig. 1. Schematic diagram of the fiber-interconnection system. Two arrays of $\mathrm{N} \times \mathrm{N}$ fibers are interconnected. $\mathrm{N} \times \mathrm{N}$ infrared beams $(\lambda=1.33 \mu \mathrm{m})$ issued from the emission array are diffracted on $\mathrm{N} \times \mathrm{N}$ elementary gratings written in the holographic plane. The elementary gratings are written by the interference of two green beams $(\lambda=514 \mathrm{~nm})$ : one with a fixed direction, and the other one with $\mathrm{N} \times \mathrm{N}$ addressable directions. The fixed-beam direction depends on the interconnection to be made. The beam-control system comprises a beam steerer and a collimating system. 
diffraction efficiency on the photothermoplastic surface material,

(4) The coupling losses of the deflected infrared beams inside the fibers must be lower than $1.5 \mathrm{~dB}$, i.e., the power coupling coefficient $\mathrm{c}$ in a monomode fiber must fit the condition

$$
c \geq 71 \%
$$

From points 1 and 2 we can deduce the power $\mathrm{Pow}_{1}$ for each writing beam:

$$
\mathrm{Pow}_{1}=\frac{1}{2} \frac{\mathrm{SE}^{2}}{\tau}
$$

wheres $=50 \mu \mathrm{J} / \mathrm{cm}^{2}$ is the sensitivity of the photothermoplastic material at $514 \mathrm{~nm}^{7}$

To avoid cross talk, the first-diffraction-order beam must not intersect the second-diffraction-order beam. Taking into account point 3 and condition (2), we find the two extreme spatial frequencies that can be written in the hol ographic plane:

$$
\begin{aligned}
& \left.\frac{1}{\Lambda}\right|_{\min }=196 \text { lines } / \mathrm{mm} \\
& \left.\frac{1}{\Lambda}\right|_{\max }=331 \text { lines } / \mathrm{mm} .
\end{aligned}
$$

We deduce that the moveable writing beam has its incidence confined in a solid angle $\Delta \varpi=\Delta \theta_{1} \times \Delta \phi_{1} \approx$ $4^{\circ} \times 4^{\circ}$.

Because the fibers used in telecommunication networks are weakly guiding, the coupling coefficient c and the mean quadratic variation $\sigma_{\phi}$ of the aberrant phase $\phi_{\mathrm{IR}}$ of the infrared beam on the entrance face of the fiber are related by ${ }^{8}$

$$
\mathrm{C}=1-\sigma_{\phi}^{2} .
$$

According to Gouy's theorem, phase $\phi_{\mathrm{IR}}$ is equal to the aberrant phase of the infrared beam just after it passes the photothermoplastic plate. We can easily estimate $\phi_{\mathrm{IR}}$ because the aberrant phase of the infrared beam incident on the photothermoplastic plane is zero. If we approximate this infrared beam by a plane wave that totally covers an el ementary cell in the deflection plane, then $\phi_{\mathrm{IR}}$ is just the phase aberration of the hologram. It is equal to $\phi_{\mathrm{IR}}=\phi_{1}-\phi_{2}$, with $\phi_{1}$ and $\phi_{2}$ being the aberrant phases of the two writing beams on the elementary cell. From Eq. (4) we conclude that

$$
\mathrm{C}=1-\sigma_{\left(\phi_{1}-\phi_{2}\right)}^{2} \geq 1-\sigma_{\phi_{1}}^{2}-\sigma_{\phi_{2}}^{2}
$$

with $\sigma_{\phi_{1}}^{2}$ and $\sigma_{\phi_{2}}^{2}$ as the mean quadratic differences of the aberrant phases of the two writing beams as computed for the surface of an elementary cell and assumed to be statistically independent. Condition (5) is highly restrictive, although the aberrant phases are computed on a very small surface, $E^{2} / N^{2}$. Indeed if, for instance, the writing wave front presents a small spherical aberration, then the slope of the phase on the border of the commutation plane will be completely different from the zero slope of the plane wave. Consequently, the aberrant phase on a small cell on the border of the commutation plane will be much larger than the average aberrant phase on the whole commutation plane. High-quality writing beams are thus required.

\section{B. Beam-Steering System}

1. Principle

The basic principle of a photor efractive beam-steering system was previously proposed and demonstrated in Ref. 9 (see Fig. 2). A plane wave uniformly illuminates an array of $\mathrm{N} \times \mathrm{N}$ optical shutters. The array is followed by a lens (not represented in Fig. 2). Opening one of shutters defines a direction for the transmitted light. There are $\mathrm{N} \times \mathrm{N}$ possibilities $(\mathrm{N} \times \mathrm{N}$ shutters) for the direction of the light. This addressing system, with no mechanical parts, is highly reliable; however, it suffers from poor transmission. The transmitted optical power is at most the input power divided by $\mathrm{N} \times \mathrm{N}$. To compensate for this low efficiency, the weak beam is amplified with two-beam coupling in a photor efractive crystal so as to obtain a deflected beam of adequate power Pow P $_{1}$ The main disadvantage of this system is its low signal-to-noise ratio. The noise comes essentially from the low contrast of the shutter array and from the amplified diffusion of light in the crystal (the beam-fanning phenomenon). We show in the next paragraph that the contrast of the shutter arrays available nowadays is sufficient for this application. We also demonstrate that the large amount of amplified scattered light coming from the photorefractive crystal results from an imperfect overlap between the beams in the crystal. In the previously proposed setup, ${ }^{9}$ the signal beams were indeed focused in the sample. To reduce the amount of noise we have modified this previously proposed scheme. Our new optical arrangement is depicted in Fig. 3.

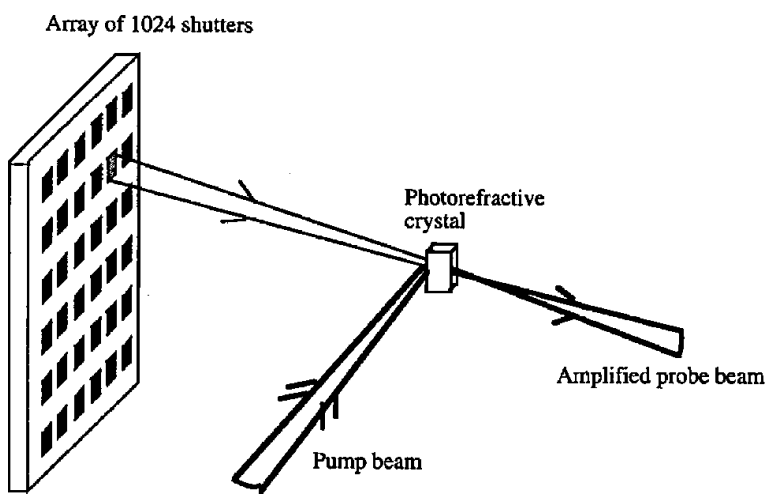

Fig. 2. Diagram of the principle scheme of a photorefractive deflector as described in Ref. 9. The opening of one shutter defines a beam direction. This beam is amplified by two-wave mixing in a photorefractive crystal. 


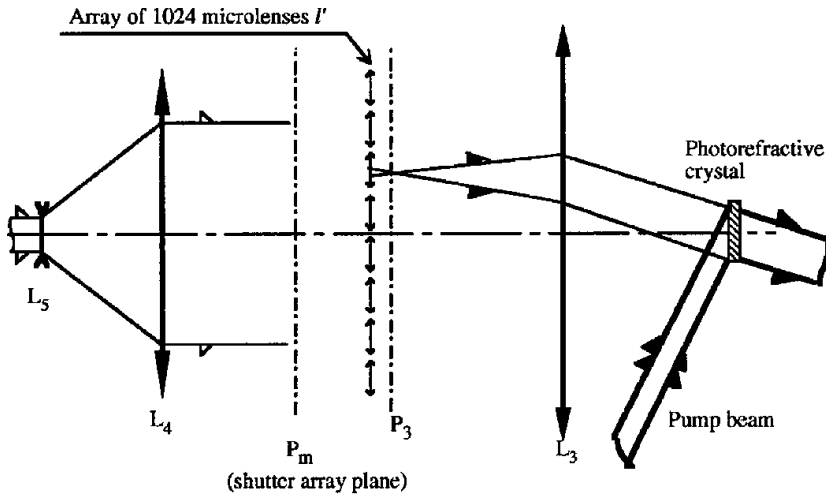

Fig. 3. Schematic diagram of the beam deflector. The object focal plane of lens $L_{3}$ is in the image plane of the microlens array $I^{\prime}$, the photor efractive crystal is in the image focal plane of $L_{3}$, and the shutter array is in plane $\mathrm{P}_{\mathrm{m}}$.

The beam is extended by means of a refractive tel escope made of a diverging doublet $L_{5}$ (focal length of $f_{5}=-50 \mathrm{~mm}$ ) and a converging doublet $L_{4}$ (focal length of $\mathrm{f}_{4}=310 \mathrm{~mm}$ ). The opened shutter in the shutter array illuminates one microlens of microlens array I'. The sel ected microl ens focuses the beam in its focal plane $P_{3}$. The focal lengths of the microlenses aref ${ }^{\prime}=13 \mathrm{~mm}$ and their pitches equal $1.09 \mathrm{~mm}$ (see Subsection 2.B.3.). $\quad \mathrm{N} \times \mathrm{N}=1024$ point sources are materialized by this process. Plane $\mathrm{P}_{3}$ is also the object focal plane for lens $L_{3}$. $L_{3}$ is the association of a converging doublet of $310 \mathrm{~mm}$ and a meniscus lens with a 400-mm focal length. The resulting focal length of this combination is $f_{3}=177 \mathrm{~mm}$. The crystal lies in the image focal plane of lens $L_{3}$. Thus, all beams from the microlenses are plane waves, and they exactly overlap each other inside the crystal. The pump-beam shape is also adjusted to exactly overlap the other beams.

The shutter array is set in the focal plane $\mathrm{P}_{\mathrm{m}}$ of the microl ens array to avoid diffraction on the photorefractive crystal. The collimating system images this crystal onto the holographic plane $\mathrm{P}_{\mathrm{c}}$. Thus, each shutter is imaged onto $P_{c}$.

One should remark that the beam-steering system is designed to address a beam of uniform power in an angular range of $\Delta \Omega=\Delta \Theta_{1} \times \Delta \Phi_{1} \approx 11^{\circ} \times 11^{\circ}$. As shown bel ow, this solid angle cannot belarger because of the angular dependence of the photorefractive gain. The total length of this beam steerer is approximately $120 \mathrm{~cm}$.

\section{Shutter Array}

Because of the low contrast of the shutter array a nonnegligible part of the transmitted light passes through the closed shutters. Let $I_{0}=I_{n, m}(0)$ be the probe-beam intensity when shutter $(n, m)$ is opened. Beam intensities $I_{i, j}(\mathrm{Co})$ that go through the closed shutters are then

$$
I_{i, j}(C o)=\frac{I_{0}}{C_{0}},
$$

where Co is the shutter-array contrast.
To write a grating in plane $P_{c}$ with a modulation ratio as high as possible, we must have the intensity $I_{0}$ be greater than the sum of intensities $I_{i, j}(\mathrm{Co})$ :

$$
\mathrm{I}_{0} \geq\left(\mathrm{N}^{2}-1\right) \frac{\mathrm{I}_{0}}{\mathrm{Co}_{0}} .
$$

With $\mathrm{N}=32$, Co must be greater than or nearly equal to 1000 . Commercially available shutter arrays provide such large contrasts, and they are sufficient for our application because the photorefractive amplifier does not modify the ratio of the transmitted beams. Indeed, as described hereafter, the exact overlapping of all beams in the photorefractive crystal makes the contrast of the array seen through the photorefractive amplifier equal to the contrast of the array without an amplifier.

\section{Microlens Array}

The microlens array is a Model MRP110 array purchased fromAeroflex. This array is made of injectionmolded methyl methacrylate whose refractive index is $\mathrm{n}_{\mathrm{m}}=1.493$ at a wavelength of $514 \mathrm{~nm}$. Its thickness is $5.18 \mathrm{~mm}$. There are $53 \times 53$ squared plano-convex microlenses. Their pitches are $\mathrm{p}^{\prime}=$ $1.09 \mathrm{~mm}$. The pitch is equal to the space between each shutter. If the focal lengths are to be increased, their curved surfaces are immersed in an index liquid, as follows:

(a) Four thickness wedges $(500 \mu \mathrm{m})$ are pasted onto the borders of the Aeroflex MRP110 array with an ultraviolet glue(EPOTEK, NOA 63).

(b) A glass plate is then pasted on the other sides of the four wedges, thus forming a cell.

(c) The sides of the cell are sealed with a highviscosity glue(EPOTEK, No. 731).

(d) A hole and a tube to use to fill the cell with an index liquid are added through the glass plate.

(e) The cell is filled while in vacuum.

The procedure is similar to that used with liquidcrystal screens. The index liquid is an epoxy resin (EPOTEK, No. 328). We measured a resulting focal length of $f_{0}=13 \pm 0.2 \mathrm{~mm}$. This microlens array is remarkably stable after construction: no recantation and no bubbles are observed even after two years.

\section{Photor efractiveAmplifier}

The photorefractive crystal compensates for loss by two-wave mixing three kinds of losses:

- The weak transmission coefficient of the shutter array $(\mathrm{t} \approx 18 \%)$,

- The total incident power on the shutter array that is divided by $\mathrm{N} \times \mathrm{N}$, and

- The geometrical losses on the diaphragms of the system (transmission factor $p_{\mathrm{g}}<1$ ).

If the total incident power at the entrance of the system is $\mathrm{Pow}_{0}=2 \mathrm{~W}$, the amplification factor $\mathrm{f}$ must 
be at least

$$
\mathrm{f} \geq \frac{1}{\mathrm{p}_{\mathrm{g}}} \frac{\mathrm{N}^{2} \mathrm{Pow}_{1}}{\mathrm{t}} \frac{\text { Pow }_{0}}{\text {. }}
$$

The power $\mathrm{Pow}_{1}$ at the exit of the photorefractive amplifier must be larger than $50 \mathrm{~mW}$ [Eq. (2)]. Consequently, the amplification factor $f$ must belarger than $140 / \mathrm{p}_{\mathrm{g}}$. One material that satisfies these two criteria is a $\mathrm{BaTiO}_{3}$ crystal. A high amplification factor is obtained along with extraordinary polarized light. One source of noise is the buildup of oscillations between the front and the rear faces of the crystal. ${ }^{10}$ This phenomenon is eliminated with antireflection-coated faces. As discussed above, the diffusion of light is also a source of noise. This noise reaches its largest value with extraordinary polarized light and is usually known as beam fanning. ${ }^{10}$ The following analysis shows how to reduce this phenomenon: Because all the waves are plane waves and overlap each other inside the sample, the evolution of the intensity versus coordinate $z$, along the propagation direction, is ${ }^{10}$

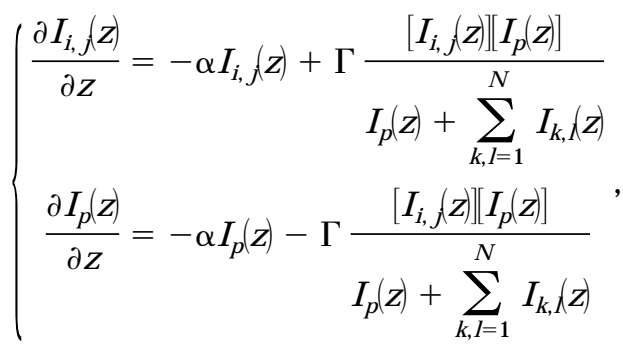

where $\Gamma$ is the photorefractive gain, $\alpha$ is the absorption, and $\mathrm{I}_{i, j}(\mathrm{z})$ represents the intensities of the light passing through the opened and the closed shutters and also accounts for noise beams. In Eqs. (9) the interactions between beams $\mathrm{I}_{\mathrm{i}, \mathrm{j}}(\mathrm{z})$ are neglected because, in the photorefractive crystal that we use, the large fringe spacings of the interference patterns between these beams make the photorefractive coupling much smaller than that between the pump beam $I_{p}(z)$ and beams $I_{i, j}(z)$, for which the fringe spacings are much smaller.

These above equations represent the generalization of two-wave mixing to multiwave mixing. They lead to

$$
\left\{\begin{array}{l}
I_{i, j}(z)=I_{i, j}(0) \frac{I_{p}(0)+\sum_{k, I=1}^{N} I_{k, I}(0)}{\sum_{k, I=1}^{N} I_{k, l}(0)+I_{p}(0) \exp (-\Gamma z)} \exp (-\alpha z) \\
I_{p}(z)=I_{p}(0) \frac{I_{p}(0)+\sum_{k, I=1}^{N} I_{k, I}(0)}{\exp (\Gamma z) \sum_{k, I=1}^{N} I_{k, l}(0)+I_{p}(0)} \exp (-\alpha z)
\end{array}\right.
$$

which can be written as

$$
I_{i, j}(z)=\left[I_{i, j}(0)\right] f\left[I_{p}, \sum_{k, l=1}^{N} I_{k, l}(0)\right] .
$$

So, if all the probe beams overlap each other inside the crystal, the amplification factor $f$ is the same for all the beams. The shutter contrast seen through the photorefractive amplifier is thus the same as the contrast without an amplifier. Moreover, the amplification factor $f$ simultaneously saturates for all probe beams and for diffusion. This simultaneous activity reduces beam fanning. ${ }^{11}$

This important reduction comes from the exact overlapping of all the beams in the crystal. Indeed, in the case of spatially distinct probe beams, the equations are those of $\mathrm{N}$ two-wave mixings. They lead to

$$
I_{i, j}(z)=I_{i, j}(0) \frac{I_{p}(0)+I_{i, j}(0)}{I_{i, j}(0)+I_{p}(0) \exp (-\Gamma z)} \exp (-\alpha z)
$$

This last relation shows that, as a result of the saturation of the photorefractive effect, the most amplified beam is the weakest one. In this case, the contrast $\mathrm{Co}^{\prime}$ of the shutter array that follows the photorefractive amplifier is lower than the contrast Co. From Eq. (11) and (12) we conclude that, to achievea beam-steering system with a large signal-tonoise ratio, all the beamlets plus the pump beam must exactly overlap each other in the crystal.

\section{Collimating System}

The collimating system is put between the beam steerer and the holographic-commutation plane $\mathrm{P}_{\mathrm{c}}$. The dimensions of this plane are $\mathrm{E} \times \mathrm{E}=10 \mathrm{~cm} \times$ $10 \mathrm{~cm}$. This plane is the image, by passage through the collimating system of the exit face of the photorefractive crystal, whose dimensions are $A \times A \approx$ $5 \mathrm{~mm} \times 5 \mathrm{~mm}$. The incident angles of the writing beams on plane $P_{c}$ range in a solid angle $\Delta \varpi=\Delta \theta_{1} \times$ $\Delta \phi_{1} \approx 4^{\circ} \times 4^{\circ}$, which is comparable with the deflection angle $\Delta \Omega=\Delta \Theta_{1} \times \Delta \Phi_{1} \approx 11^{\circ} \times 11^{\circ}$ of the beamsteering system. Thus, the transversed magnification of the collimating system is $M=E / A=20$, and the convergence ratio is $1 / \gamma=\Delta \Theta_{1} / \Delta \theta_{1} \approx 3$. For a centered optical system with an object and an image medium of same refractive indices we have $M \gamma=1$ (Lagrange-Helmholtz relation). The only way to break this relation and to achieve $\mathrm{M}=20$ and $1 / \gamma \approx 3$ is to design an off-axis system. Such a scheme is shown in Fig. 4. The system is composed of a microlens array I inserted between two lenses, $L_{1}$ and $L_{2}$. Lens $L_{1}$ is similar tolens $L_{3}$ (see Fig. 3 ). It is placed after the crystal and it forms a refractive telescope with $L_{3}$. It images, in a $4 f$ system with a unit-magnification ratio, the point sources of the microlens foci. Theaxis of the movable beam, after it passes through lens $L_{1}$, is parallel to $\left\langle\mathrm{O}^{\prime} \mathrm{O}\right\rangle$ and is focused in the focal plane, $P_{1}$ of lens $L_{1}$. A shutter array $(32 \times 32)$ is put in plane $P_{1}$. As described 


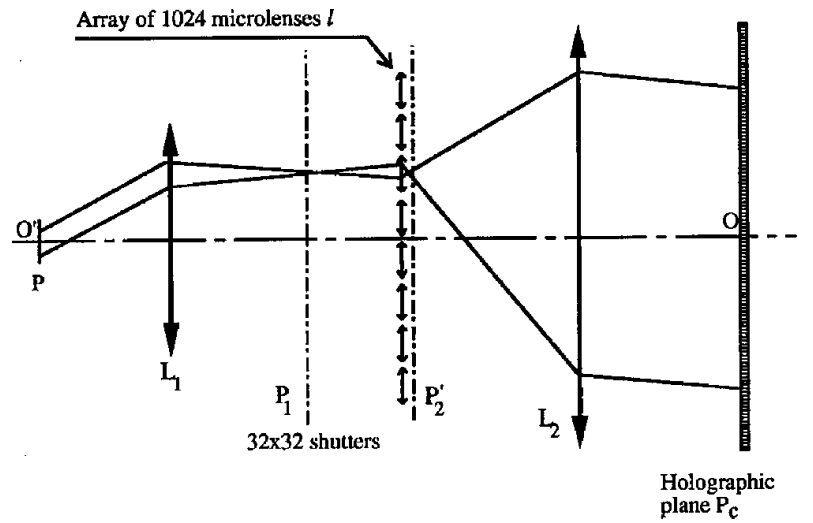

Fig. 4. Sketch of the collimating system. $P_{1}$ is in the object focal plane of lens $L_{1}$. The holographic plane $P_{c}$ is in the image focal plane of lens $L_{2}$. $P$, the exit face of the photorefractive crystal; $\mathrm{O}^{\prime} \mathrm{O}$, the axis of the collimating system.

above, the M RP 110 microlenses were purchased from Aeroflex. Their pitches were confirmed $(1.09 \mathrm{~mm})$, and their focal lengths were measured $(3.2 \mathrm{~mm})$. One of the microlenses (corresponding to one selected direction) increases the optical aperture of the beam that is focused in plane $\mathrm{P}_{2}^{\prime}$. $\mathrm{P}_{2}^{\prime}$ is also the object focal plane of lens $L_{2}$ that maintains the plane-wave structure at the exit of the collimating system. $P_{c}$ is in the image focal plane of $L_{2}$. The writing beam that has variable incidence angles is always incident at the same place on $\mathrm{P}_{\mathrm{c}}$ and illuminates the same useful area on the photothermoplastic plane. The required focal distance $f_{2}$ for lens $L_{2}$ is $f_{2}=488 \mathrm{~mm}$. We have designed this lens to fit the wave-front quality requirements. We have also taken care to leave enough space between that lens and the commutation plane that the infrared beams will be incident on the commutation plane. This lens results from the association of four elementary lenses, as shown in Fig. 5. The microlens array is glued onto the first lens (on the far-left side of Fig. 5). One should remark the important volume of this system (a length of approximately $1 \mathrm{~m}$ and a diameter of $\mathrm{D}_{2} \geq 250 \mathrm{~mm}$ ). The total distance between the microlens array and the holographic-commutation plane is approximately $140 \mathrm{~cm}$. The coupling losses resulting from objective $L_{2}$ must be lower than 30\% [Eq. (1)]. The maximum slopes of the aberrant phase as functions of the point-source positions have been computed and are shown in Fig. 6 . The maximum slope is $2.2 \times$ $10^{-5} \mathrm{rad}$ for the writing beam that has a variable

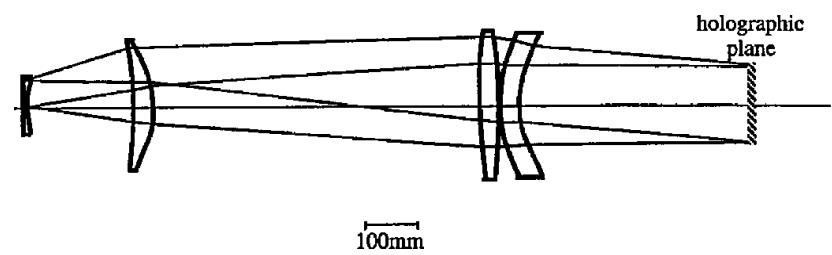

Fig. 5. Diagram of the exact design of lens $L_{2}$. The microlens array is designed to be glued onto the first lens on the left-hand side.

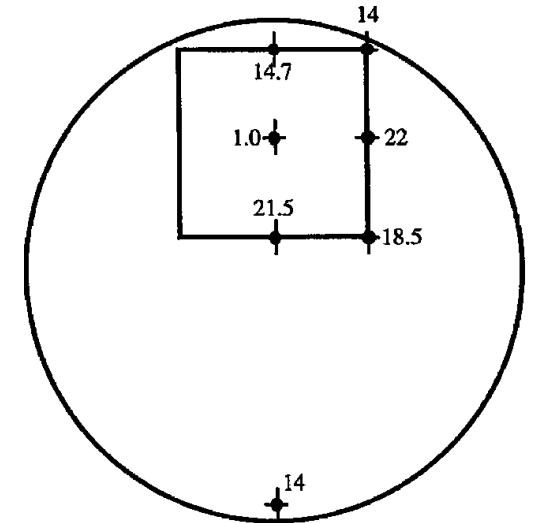

Fig. 6. Maximum phase slope $(\mu \mathrm{rd})$ of the wave incident upon the holographic plane as a function of the source point. These sources are located on the first diopter of lens $L_{2}$. The source points of the writing beam issued from the beam-steering system are contained in the square and its edges. Point 14, outside the square, represents the fixed writing beam. The microlenses on the sides of the array induce the highest aberrations.

incidence. The maximum slope of the aberrant phase for the fixed writing beam is $1.4 \times 10^{-5}$ rad. The sum of these two values is lower than $5 \times 10^{-5} \mathrm{rad}$, which corresponds to $\sigma_{\phi}^{2}<0.3 \%$. The collimating system has been entirely designed and computed, but it has not been manufactured because of its cost.

\section{Beam-Steering System Characterization}

A. Microlens Array

In the following subsection, several characteristics of the MRP 110 microlenses are analyzed: (1) the quality of their surfaces, (2) their focal lengths, and (3) their spherical aberrations.

\section{SurfaceQuality}

For this study, the microlens array is illuminated by a plane wave from an argon laser $(\lambda=514 \mathrm{~nm})$. The light is incident upon the curved faces of the microlenses. We select the beam from one microlens by putting a pinhole(1-mm diameter) at the focal point of this microlens. The front face of the microlens is imaged onto a CCD array. Two kinds of defects are observed: (1) Scratches, whose widths are much smaller than the size of the microlens illuminating the commutation plane (these imperfections are not an inconvenience for the system); and (2) craters, whose highest dimensions are $40 \mu \mathrm{m}$ and whose depths are of the order of $1 \mu \mathrm{m}$ (this last quantity is measured with a Mach-Zehnder interferometer that is described in Subsection 3.A.3. The surface quality of an individual microlens is better at the center and inside a squared $0.7 \mathrm{~mm} \times 0.7 \mathrm{~mm}$ pupil. The craters are sparse and thin in this region and are usually not detected on the corresponding interferograms.

\section{Focal-Length Measurement at $\lambda=514 \mathrm{~nm}$}

A parallel-polarized beam of light is incident on the curved face of one microlens. That curved face is imaged onto a CCD array with a microscope objective. 
This objective and the CCD array are then moved together by the same amount to image the focal point of the microlens onto a CCD camera. This displacement represents the difference between the image plane of the curved face observed through the microlens and the focal plane of the microlens. Consequently, it is the focal length of the microlens. Different measurements for many microlenses give a mean focal distance of $\mathrm{f}_{0}=3.2 \mathrm{~mm}$. The fluctuations $( \pm 20 \mu \mathrm{m})$ about this valueareincluded in the measure ment uncertainties.

\section{Aberration Studies}

A shadow experiment yielded an estimate of the phase defects. The light source is an Ar laser $(\lambda=514 \mathrm{~nm})$ and illuminates the entire microlens array. One microl ens is selected by the placement of a pinhole (1-mm diameter) at the focal point of the microlens. A diffusing screen is set $150 \mathrm{~mm}$ after the array to observethe conical projection of the transmitted light; the diffusing screen is then imaged onto a CCD array. This arrangement reveals the local variations in the phase. The slow variations, e.g., spherical aberrations, are not detected with this experiment. However, various defects are observed: dark lines al ong the microl enses (interferometry reveals that the order of the phase variation is approximately $\lambda / 10$ and is negligible for the application) and stretches along a diagonal.

A Mach-Zehnder interferometer was used to obtain interferograms of the microlenses. The microlens array is inserted into one arm of the interferometer. One microlens with a pinhole at the focal point of the microlens is selected. The interference pattern is observed on the curved face of the microlens. As shown in Fig. 7, some microlenses are astigmatic, and their interference rings are elliptical. This interferogram corresponds to a microlens with the phase defect seen as a dark line in the shadow experiment. Different profiles passing through the centers of the rings are taken, and they give the positions of the

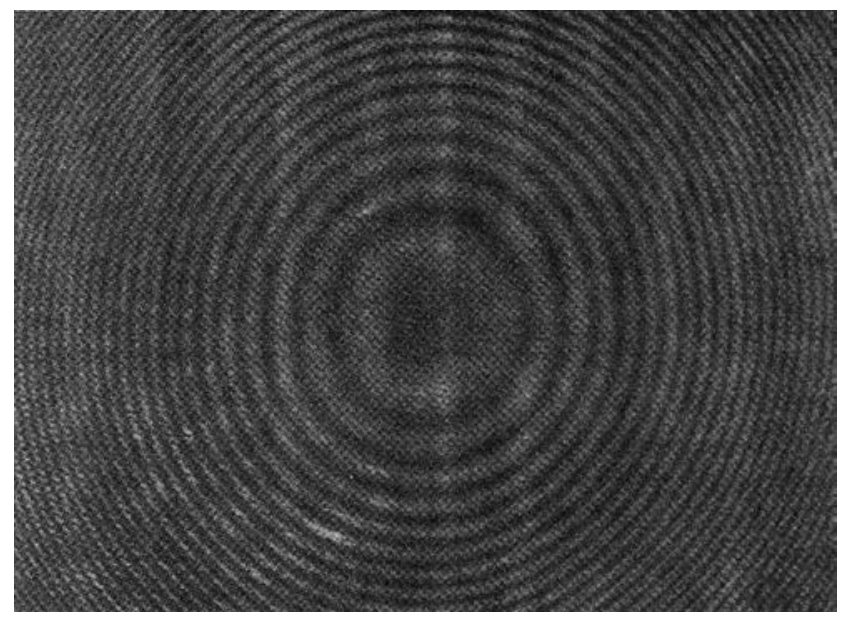

Fig. 7. Interferogram of the center $(\approx 0.6 \mathrm{~mm} \times 0.4 \mathrm{~mm})$ of a microlens that presents astigmatism. The interference rings are elliptical.

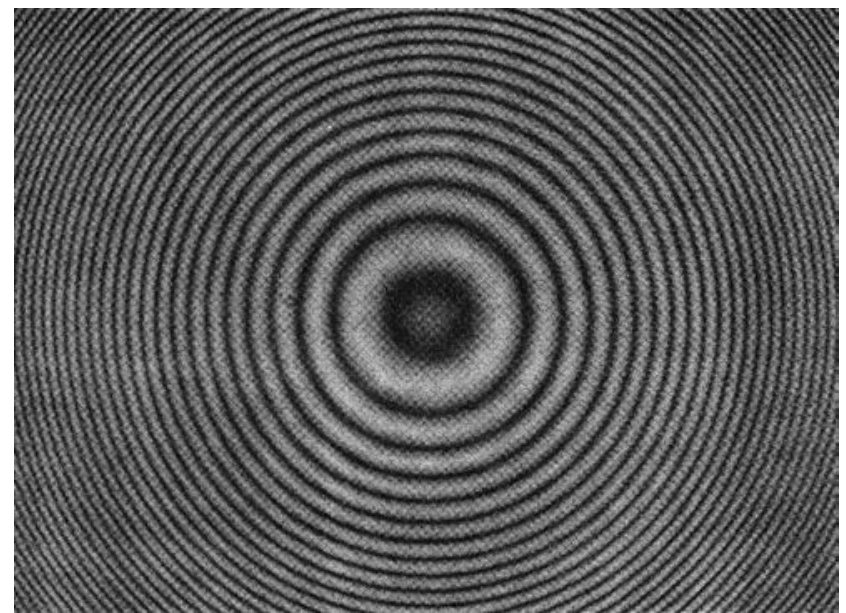

Fig. 8. Interference pattern of the center of a microlens (the same approximate size as for Fig. 7) that is not astigmatic. The rings are circular, and the corresponding losses are negligible.

minimum intensities as a function of the interference order. From this curve, the phase defect is deduced and was found to be lower than $0.5 \lambda$.

All the microlenses do not present the same defects. As an example, Fig. 8 shows the interferogram of another microlens for which the rings are quite circular. For such lenses the only aberration to take into account is the spherical aberration, because all microlenses are illuminated by the same beam at normal incidence. The phase of the transmitted wave at the exit of a microlens is developed by means of the Zernicke polynomials. ${ }^{12}$ The coupling coefficient $\mathrm{c}$ in a monomode fiber of the reception array is numerically computed as a function of the elementary gratings written in $\mathrm{P}_{\mathrm{c}}$. The most important coupling losses are for the elementary gratings written on the sides of $P_{c}$. If a square, $0.68 \mathrm{~mm} \times 0.68 \mathrm{~mm}$ pupil at the entrance of a microlens is used, the induced coupling loss remains at approximately $\sigma_{\phi}^{2} \approx 6 \%$. The coupling losses in this case are thus negligible. In the center of the $53 \times 53$ microlens array most lenses are acceptable. However, we did not check whether a square containing $32 \times 32$ microlenses with negl igible defects could be found.

In summary, the main parameters, such the focal length, the surface quality, the aberrations, and the corresponding coupling losses induced by these lenses, have been studied. The calculations of the coupling losses coming from the aberrations of the microlenses show that a $0.68 \mathrm{~mm} \times 0.68 \mathrm{~mm}$ pupil can be used, resulting in acceptable losses. These microlenses can thus be used in the collimating system.

The aberrations of the immersed microlens array used for the beam-steering system (with a focal length of $\mathrm{f}_{0}=13 \mathrm{~mm}$ ) are lower than those in the initial MRP110 array. The aberrations are reduced by a factor of

$$
\frac{\left(\mathrm{n}_{\mathrm{m}}-\mathrm{n}_{\mathrm{air}}\right)}{\left(\mathrm{n}_{\mathrm{m}}-\mathrm{n}_{\text {liq }}\right)} \approx 4,
$$


where $\mathrm{n}_{\text {liq }}$ is the refractive index of the EPOTEK 328 epoxy resin $\left(n_{\text {liq }} \approx 1.41\right.$ at $\left.\lambda=514 \mathrm{~nm}\right)$. Thus, it is clear that these aberrations are totally negligible and that it is not useful to measure them.

\section{B. Photorefractive Crystal}

The photorefractive amplifier is a barium titanate $\left(\mathrm{BaTiO}_{3}\right)$ crystal. The signal-to-noise ratio $\mathrm{R}$ of the crystal must be such that the power of the amplified beam is higher than the noise in the $(\mathrm{N} \times \mathrm{N})-1$ directions where the beams are cut off:

$$
\mathrm{R} \geq\left(\mathrm{N}^{2}-1\right) \approx 1000 .
$$

The dimensions of the crystal along the crystallographic axes are $\hat{a} \times \vec{b} \times \hat{c}=3.63 \mathrm{~mm} \times 5.22 \mathrm{~mm} \times$ $5.24 \mathrm{~mm}$, respectively. This crystal sample was bought from the Institute of Physics, Chinese Academy of Sciences, Beijing, China. Its absorption coefficient is $\alpha=0.76 \mathrm{~cm}^{-1}$ at $\lambda=514 \mathrm{~nm}$; its trap density, measured through a two-wave-mixing arrangement, 10 is $\mathrm{Na}=2 \times 10^{22} \mathrm{~m}^{-3}$. The two incident faces â are $\lambda / 8$ polished so that the coupling losses in a monomode fiber in the reception array are numerically computed to be $\sigma_{\phi}^{2} \approx 0.06 \%$. These two faces are also antireflection coated to el iminate oscillations between the front and the rear faces. The reflection coefficient of the coating should ensure that the product of the gain times the losses is lower than 1 . This leads to a reflection coefficient lower than $4 \%$. We measured it to be lower than $1 \%$ at $\lambda=514 \mathrm{~nm}$. We have verified that the oscillations do not build up with this coating. The quality of the transmitted wave was measured with a Mach-Zehnder interferometer. The interference pattern between the transmitted wave and a reference wave is recorded on a CCD array. This pattern shows that the phase is not uniform. These variations of the phase come from inhomogeneities inside the crystal bulk and from crystal-surface defects. The volume inhomogeneities are small enough and do not induce significant coupling losses. This is not the case for defects at the surface that result from the rotation of domains during the polishing process. The $\hat{c}$ axes of the rotated domains are now perpendicular to the entrance face and so to the $\hat{c}$ axis of the crystal. The small vol ume of these domains does not affect amplification, but it is sufficient to alter the wave front of an extraordinary polarized beam propagating through the crystal. Another $\mathrm{BaTiO}_{3}$ sample from the same origin was studied. This sample was polarized after it was polished and before it was antireflection (by Virgo Optics, U.S.A.) coated, and it does not present any microdomains. Although the photorefractive gain of the sample is too low to be useful in our setup, it demonstrates that high-quality samples can be obtained. Interferometric measurements indicate that the coupling losses induced by such a sample are negligible.

\section{Beam Steerer}

The characterization of the beam-steering system consists of four parts:
(1) The phase quality of the transmitted wave;

(2) The amplification factor versus the deflected direction;

(3) The energetic balance of the system;

(4) The signal to noise ratio.

\section{Phase Quality of the Transmitted Wave}

Because the beamlets cover only a small surface of lenses $L_{1}, L_{2}$, and $L_{3}$, the aberrations introduced by these lens are negligible. As discussed above, the aberrations introduced by the immersed microlenses are al so negligible. Most aberrations thus come from the photorefractive crystal. The influence of these aberrations on the deflected beam was measured with a Mach-Zehnder interferometer. Theamplified wave interferes with a spherical reference wave, and the resulting interference pattern is recorded on a CCD array placed in an image plane of the photorefractive crystal. The interference pattern reveals aberrations larger than $\lambda$, even when the pump beam is cut off. These defects come from the tilted domains that appear after polishing. With the second crystal sample that is repolarized after it is polished, no such aberrations are observed on the interferograms. This demonstrates that the tilted domains induce nonnegligibleaberrations. Unfortunately, the amplification factor of this other sample was too low for the application.

\section{Amplification Factors}

The incident angle for the pump beam is $55^{\circ}$. The average probe-beam incident angle, corresponding to a microlens in the middle of the $32 \times 32$ array, is $20^{\circ}$. An amplification factor of $6000\left(\Gamma=24 \mathrm{~cm}^{-1}\right)$ is reached if a microlens in the center of the microlens array is selected. The uniformity of the amplification is studied through the selection of one microlens in the center of the microlens array or of each of the four microlenses that are in the corners of the $32 \times 32$ array. The results of the uniformity evaluation are listed in Table 1. The measurements of the gain $\Gamma$ and of amplification factor $f_{1}$ are conducted with a high pump-to-probe beam intensity ratio.

Table 1. Measurements of Amplification Factors $f_{1}$ and $f_{2}$ as Functions of the Positions of the Illuminated Microlenses ${ }^{a}$

\begin{tabular}{lccc}
\hline Beam Position & $\begin{array}{c}\text { Gain } \Gamma \\
\left(\mathrm{cm}^{-1}\right)\end{array}$ & $\begin{array}{c}\text { Amplification } \\
\text { Factor } \mathrm{f}_{1}\end{array}$ & $\begin{array}{c}\text { Amplification } \\
\text { Factor } \mathrm{f}_{2}\end{array}$ \\
\hline Center & 24 & 6600 & 1300 \\
Down right & 21 & 2000 & 1200 \\
Up right & 20 & 1700 & 1100 \\
Up left & 20 & 1900 & 1200 \\
Down left & 20 & 1900 & 1400 \\
\hline
\end{tabular}

$\mathrm{a}_{1}$ and $\Gamma$ were measured through the use of a high pump-to-probe beam intensity ratio, and $f_{1}$ varies by more than a factor of $3 . f_{2}$ was measured under photorefractive-saturation conditions and is approximately constant. 
In this case, amplification factor $f_{1}$ varies by more than a factor 3. If one takes into account the Gaussian shape of the beam illuminating the microlens array, the amplified-beam intensities vary by more a factor of 7. The measurements of amplification factor $f_{2}$ are obtained when the pump-to-probe beam intensity ratio is decreased so that it reaches the saturation of the photorefractive effect. By this means, amplification is smoothed and is more uniform: $f_{2}$ varies by no more than $\pm 10 \%$, which corresponds to variations of the amplified beams by a factor of 3 only.

\section{Energetic Balance}

The total power $(600 \mathrm{~mW})$ at the entrance of the beam steerer is divided in two parts. The first, part 1, illuminates the microlens array, while the second, part 2, corresponds to the pump beam. The power of the amplified probe beam is measured as a function of the intensity ratio, part 1 /part 2 . The results are reported in Table 2. The maximum deflected power is $32 \mathrm{~mW}$. For this value, the efficiency,

$$
\eta=\frac{\text { power of the deflected beam }}{\text { total power }}
$$

of the beam steerer is 5.3\%. In the case of photorefractive saturation, the efficiency is $2.5 \%$ (row 7 in Table 2). It must be noted that the efficiency is higher than required (a deflected power of $50 \mathrm{~mW}$ is needed for an incident power of $2 \mathrm{~W}$, i.e., $\eta=2.5 \%$ ).

To demonstrate the performance of the beam steerer, one conducts the following procedure: If all the light power goes through a microlens (an infinite intensity ratio, part 1 /part 2), the maximum deflected power is $21 \mu \mathrm{W}$, which yields $\eta=0.0035 \%$. This value must be compared with the maximum deflected power (32 $\mathrm{mW}$ ) when photor efractive amplification is used. The efficiency of the system is increased by a factor of $\mathbf{1 5 0 0}$ with the photorefractive

Table 2. Efficiency $\eta$ of the System for Different Pump-to-Probe Beam Intensity Ratios ${ }^{a}$

\begin{tabular}{cccc}
\hline $\begin{array}{c}\text { Pump Power } \\
(\mathrm{mW})\end{array}$ & $\begin{array}{c}\text { Amplified } \\
\text { Probe-Beam Power } \\
(\mathrm{mW})\end{array}$ & $\begin{array}{c}\text { Amplification } \\
\text { Factor } \mathrm{f}\end{array}$ & $\begin{array}{c}\text { Efficiency } \eta \\
(\%)\end{array}$ \\
\hline 340 & 3.5 & 5800 & 0.6 \\
309 & 7.9 & 4900 & 1.3 \\
250 & 15.5 & 4700 & 2.6 \\
190 & 23.5 & 5000 & 4 \\
130 & 32 & 5100 & 5.3 \\
80 & 30 & 4300 & 5 \\
32 & 15 & 1880 & 2.5 \\
21 & 12 & 420 & 2 \\
7 & 3.8 & 140 & 0.6 \\
\hline
\end{tabular}

aThe intensity ratio comprises parts 1 , which illuminates the microlens array, and 2, the pump beam, of the total power of $600 \mathrm{~mW}$ at the entrance of the beam steerer. When no crystal is used and all power goes through the single arm, the efficiency $0.00004 \%$. If one part of the incident beam is used as a pump beam and a crystal is inserted, the efficiency, under photorefractive saturation, increases to $2.5 \%$ (see row 7 ). crystal. In consequence, the system reduces the total power for switching by a factor of 1500 .

\section{Signal-to-NoiseRatio}

Optical noise is defined as the diffused-light intensity in the direction of a closed shutter when another shutter is opened. The contrast of the shutter array being high, this source of noise is negligible. For the demonstration, in place of these shutters, we use a cardboard mask. The noise is due essentially to beam fanning. The measurement of the noise is conducted with the worst possible configuration: A microlens in the center is illuminated. The amplification factor is thus at its maximum (6000) and produces a beam-fanning process that is as high as possible. The intensity is also adjusted so as to avoid pump-beam saturation. Theamplified signal is measured with a detector in the image plane of the microlens focal plane. We analyze the noise by moving the detector to other focal points that correspond to microlenses that are not illuminated. Noise is at the maximum for microlenses closest to the center. The signal-to-noise ratio is 5500 for the nearest vertical microlenses and 7300 for the nearest horizontal microlenses. The noise is greater al ong the vertical axis because of conical diffraction. All these values are higher than the maximum value of 1000 required for the system. Even better ratios could be obtained if the intensity pump-to-probe ratio were decreased or if spatial filters were added in the microlenses image plane.

\section{Conclusion}

A beam-control system for use when gratings are written on a holographic plane has been thoroughly studied. The total length of this system is $260 \mathrm{~cm}$. The whole system is composed of two elements: a beam steerer that directs a beam along one possible direction among $\mathrm{N}^{2}$ directions and a collimating system that adapts the section and the incidence of the deflected beam to the holographic plane.

We found the foll owing important conclusions:

(1) Photorefractive crystals are well adapted to low-noise beam-steering systems. The surface quality of the sample we used was too poor; however, as seen with a second sample, surface-quality defects can be avoided. The signal-to-noise ratio of the beam steerer is larger than 5500. This high ratio is obtained when all the beams inside the crystal are overlapped and an antireflection coating is deposited on the faces. The deflection efficiency when such a crystal is used is higher than $2.5 \%$, whereas, at the maximum, it is equal only to $4 \times 10^{-5}$ when without a photorefractive crystal. The power of the deflected beam versus its direction varies by less than a factor of 3.

(2) The required coupling coefficient in monomode fibers imposes negl igible aberrations on the two writing beams. 
We believe that point 2 is the most restrictive. It is in order to achieve such low coupling losses that we have been obliged to design the large and expensive objective label ed $\mathrm{L}_{2}$. Moreover, the size of the whole system makes its exploitation unrealistic. This point becomes still more critical when one considers that, in principle, a system able to connect 1024 input fibers with 1024 output fibers should consist of two holographic planes, each with its own addressing system. A possible way to avoid this duplication is to split the hologram array into two parts, corresponding to two different arrays of 512 fibers. In this case (by the addition of an intermediate mirror) the addressing system that we have studied could permit a twodeflection-stage interconnection system with 512 input ports and 512 output ports to be addressed.

This study underlines all the advantages of the use of dynamic couplers based on photorefractive doublephase conjugators. ${ }^{13}$ With such dynamic couplers a beacon beam issued from the target monomode fiber attracts the incident beam toward the fiber, thus ensuring large coupling efficiencies. The coupling efficiency is practically independent of the quality of the incident infrared beam. Therefore low-quality writing beams (i.e., spherical waves) could be used to record gratings in the hol ographic-commutation plane. These dynamic couplers must therefore considerably simplify the design of the beam-control system.

\section{References}

1. J . W. Goodman, A. R. Dias, and L. M. Woody, "Fully parallel, high-speed incoherent optical method for performing discrete Fourier transforms," Opt. Lett. 2, 1-3(1978).

2. M. Yamaguchi, T. Matsunaga, S. Shirai, and K. Yukimatsu,
"Analog free-space optical switch structure based on cascaded beam shifters," IEICE Trans. Commun. E77-B(2), 163-173 (1994).

3. P. Gravey, L. Bonnel, and J . Y. Moisan, "Preliminary evaluation of a 144144 holographic interconnection system," IEE Conf. Pub. (London) 311, 195-199 (1989).

4. H. Yamazaki and M. Yamaguchi, "Holographic optical switching using a ferroelectric liquid crystal spatial light modulator," paper presented at the International Meeting on Photonic Switching, Minsk, Belarus, 1-3 J uly 1992, paper 3D4.

5. J . Y. Moisan, P. Gravey, R. Lever, and L. Bonnel, “Improvement of photothermoplastic devices for their application to optical switching,"Opt. Eng. 25, 151-156 (1986).

6. P. Mathey, G. Pauliat, and G. Roosen, "Low-noise two-wave mixing deflector," paper presented at the OSA Topical Meeting on Photorefractive Materials, Effects, and Devices, Kiev, Ukraine, 11-13 August 1993.

7. H. M. Smith, ed., Holographic Recording Materials (SpringerVerlag, New York, 1977), Chap. 6.

8. A. W. Snyder and J. D. Love, eds., Optical Waveguide Theory (Chapman and Hall, London, 1983), Chaps. 13 and 20.

9. D. Rak, I. Ledoux, and J . P. Huignard, "Two-wave mixing and energy transfer in $\mathrm{BaTiO}_{3}$. Application to laser beamsteering,"Opt. Commun. 49, 302-306 (1984).

10. P. Günter and J . P. Huignard, Photorefractive Materials and their Applications I, Vol. 61 of the Topics in Applied Physics Series (Springer-Verlag, Berlin, 1989); Photorefractive Materials and their Applications II, Vol. 62 of the Topics in Applied Physics Series (Springer-Verlag, Berlin, 1989).

11. M. Segev, Y. Ophir, and B. Fisher, "Non-linear multi-two-wave mixing, the fanning process, and its bleaching in photorefractive media," Opt. Commun. 77, 265-274 (1990).

12. M. Born and E. Wolf, Principles of Optics (Pergamon, New York, 1980).

13. N. Wolffer and P. Gravey, "High-quality phase conjugation in a double phase conjugate mirror using InP:Fe at $1.3 \mu \mathrm{m}$," Opt. Commun. 107, 115-119(1994). 